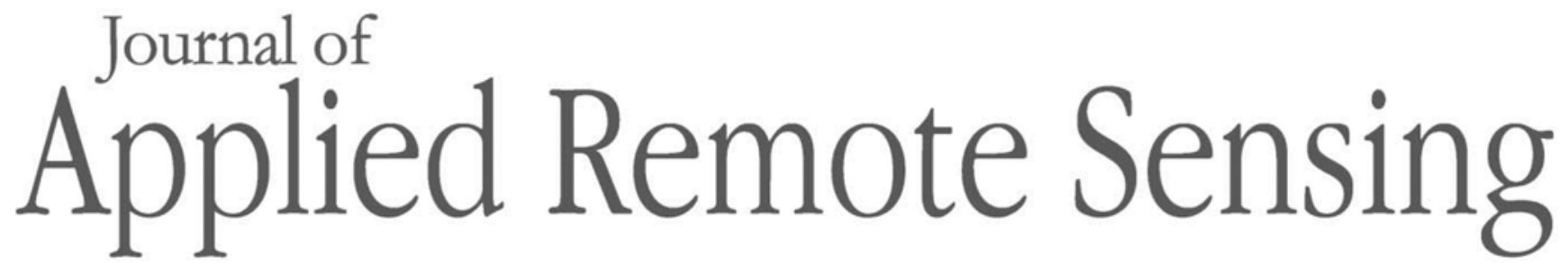

RemoteSensing.SPIEDigitalLibrary.org

\title{
Array error calibration methods in downward-looking linear-array three-dimensional synthetic aperture radar
}

\author{
Weixian Tan \\ Pingping Huang \\ Kuoye Han \\ Qi Liu \\ Xueming Peng
}




\title{
Array error calibration methods in downward-looking linear-array three-dimensional synthetic aperture radar
}

\author{
Weixian Tan, ${ }^{\mathrm{a}, *}$ Pingping Huang, ${ }^{\mathrm{a}}$ Kuoye Han, ${ }^{\mathrm{b}}$ Qi Liu, ${ }^{\mathrm{c}}$ and \\ Xueming Peng ${ }^{d}$ \\ anner Mongolia University of Technology, College of Information Engineering, \\ Hohhot 010051, China \\ ${ }^{\mathrm{b}}$ China Electronics Technology Group Corporation, Information Science Academy, \\ Beijing 100098, China \\ ${ }^{c}$ China Institute of Water Resources and Hydropower Research, Beijing 100038, China \\ ${ }^{\mathrm{d}}$ Carestream Health, Inc., Global RD Centre (Shanghai), Shanghai 201206, China
}

\begin{abstract}
In order to achieve high-precision three-dimensional (3-D) imaging with an airborne downward-looking linear-array 3-D synthetic aperture radar (LA-3D-SAR), a uniform virtual antenna array can be obtained by aperture synthesis of the cross-track sparse multiple-inputmultiple-output array. However, the actual 3-D imaging quality is unavoidably degraded by array errors such as the multichannel amplitude-phase errors due to the nonideal antenna characteristics, and the virtual element position errors due to vibrations and motion measurement deviations. We investigate the effects of these errors on the forms and the degrees of image quality degradation and consider the use of corresponding calibration methods to eliminate the effects of errors. For the multichannel amplitude-phase errors, the target response is subject to an integrated sidelobe level increase introduced by the phase error, which can be calibrated based on external (parallel or point target) calibrators, as proposed in the paper. For the virtual element position errors, they mainly the result of contrast degradation and noise in the image along the cross-track direction and have little impact on the range and along-track directions. The imaging performance is more sensitive to the error component in the height direction as compared to other components, the precision requirement of which should be established as the calibration reference. A calibration method based on time-divided active calibrators is proposed to estimate and correct the virtual element position errors. Both numerical simulations and real data experiments have shown the validity of the analyses as well as the effectiveness of the proposed calibration methods. (c) The Authors. Published by SPIE under a Creative Commons Attribution 3.0 Unported License. Distribution or reproduction of this work in whole or in part requires full attribution of the original publication, including its DOI. [DOI: 10.1117/1.JRS.10.025010]
\end{abstract}

Keywords: array error calibration; three-dimensional imaging; downward-looking linear-array synthetic aperture radar; synthetic aperture radar.

Paper 15827 received Nov. 25, 2015; accepted for publication Apr. 7, 2016; published online May 2, 2016.

\section{Introduction}

In the last decade, an increased interest has been devoted to three-dimensional synthetic aperture radar (3D-SAR), ${ }^{1}$ because of its capability to generate 3 -D radar images by retrieving the reflectivity and real 3-D localization information of the scattering objects. Among them, the downward-looking linear array SAR (LA-3D-SAR) ${ }^{2}$ with a linear antenna array [e.g., a sparse multiple-input-multiple-output (MIMO) array ${ }^{3-8}$ is usually adopted to reduce the complexity of the linear array antenna, where multiple antennas are used for transmitting while others are for receiving.] deployed under the wings of airplanes as the most feasible configuration for topographical survey application. With the rectilinear motion and the downward-looking working mode, LA-3D-SAR synthesizes a 2-D plane array to achieve 3-D imaging of illuminated

*Address all correspondence to: Weixian Tan, E-mail: wxtannm@vip.163.com 
areas and overcomes the layover and shadowing problems existed in 2-D SAR. Until now, two LA-3D-SAR systems have been developed, ${ }^{9,10}$ and some 3-D image formation algorithms have been proposed. ${ }^{11-16}$

As a multichannel coherent system, LA-3D-SAR is particularly susceptible to channel inconsistency, which will cause geometric distortion, loss of resolution, decrease in image contrast, spurious targets, and a reduction in signal-to-noise ratio (SNR). ${ }^{17}$ However, under realistic operational conditions, the antenna elements are subject to array errors such as multichannel amplitude-phase errors and antenna position errors. These errors come from the nonideal antenna characteristics, vibrations, and motion measurement errors, which may cause channel inconsistency that is large enough to deteriorate the 3-D imaging quality, especially for the LA-3D-SAR working in a high frequency band (e.g., X, Ka band). Thus, the study on the high-precision amplitude-phase consistency of LA-3D-SAR is anticipated across all MIMO channels.

In Refs. 18 and 19, the error effects and calibration methods for channel inconsistency based on the deterministic patterns of the wings' oscillation and the flight attitude variations are investigated. In this paper, we focus on two other kinds of array errors: multichannel amplitude-phase errors and random virtual element position errors, neither of which has received sufficient attention in the current research. According to their mechanisms, different error models are established, and their effects on the LA-3D-SAR images are studied. A close examination of the effects of the errors with regard to their characteristics, the levels of severity, and the relevant calibration methods is presented to specify the tolerable levels, which is important for the system design and optimization. Specific calibration methods are proposed to deal with the array errors, which are verified by either the ground-based experiment data or some numerical simulations.

The remaining sections are organized as follows. The imaging principle of LA-3D-SAR is first reviewed in Sec. 2, where the geometric and signal models are given. In Sec. 3, with different error models, the error effects of the multichannel amplitude-phase errors and the virtual element position errors are analyzed. In Sec. 4, different calibration methods based on external calibrators are proposed to deal with the errors, respectively. The ground-based experiment and numerical simulation verification are presented in Sec. 5. This paper is concluded in Sec. 6.

\section{Imaging Principle of Downward-Looking LA-3D-SAR}

As shown in Fig. 1, the radar is mounted on an airplane flying at altitude $\mathrm{H}$ with the velocity $V_{a}$. Let the $X$-, $Y$ - and $Z$-axes denote the cross-track, along-track, and height directions, respectively. In cross-track, a sparse MIMO array with $M_{\mathrm{T}}$ transmitters and $M_{\mathrm{R}}$ receivers is adopted to form

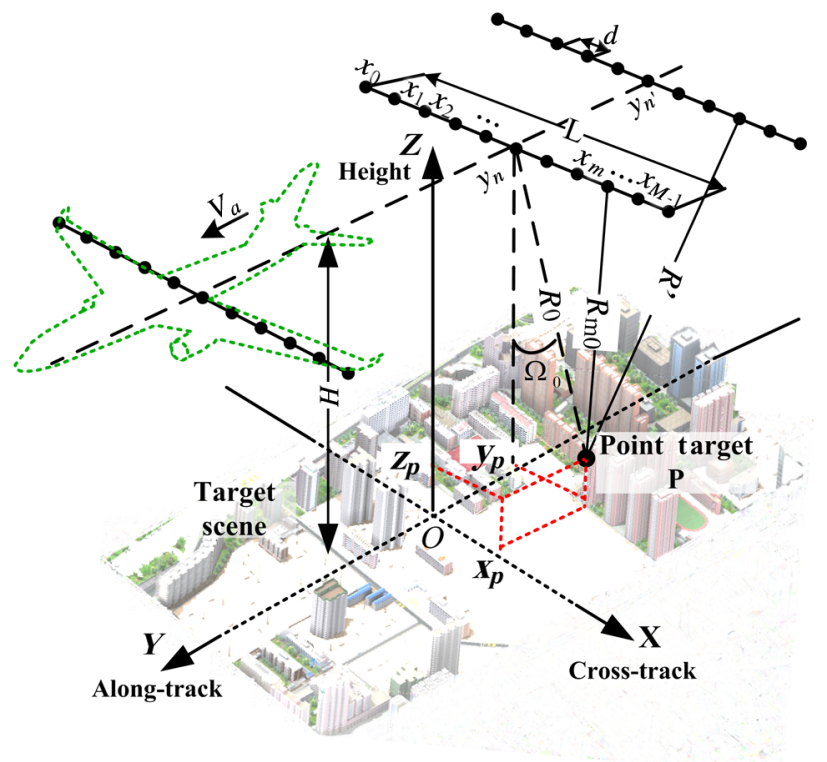

Fig. 1 Imaging geometry of downward-looking LA-3D-SAR. 
a uniform virtual array, which has $M=M_{\mathrm{T}} M_{\mathrm{R}}$ virtual elements and total length $L=(M-1) d$, where $d$ represents the element spacing. The position of the $m$ 'th virtual element is $\left(x_{m}, y_{n}, H\right)$, where $y_{n}$ represents the along-track spatial sample coordinate. The illuminated scenario is located at the nadir area of the platform, and for a point target $P$ with reflectivity $\sigma_{p}$ located at $\left(x_{p}, y_{p}, z_{p}\right)$ in the 3-D target support band $\Omega$, the instantaneous target-to- $m$ 'th virtual element distance is

$$
R^{\prime}\left(x_{m}, y_{n} ; P\right)=\sqrt{\left(x_{m}-x_{p}\right)^{2}+\left(y_{n}-y_{p}\right)^{2}+\left(H-z_{p}\right)^{2}} .
$$

Assuming that orthogonal pulses with pulse length $T_{p}$, frequency bandwidth $B$, and carrier frequency $f_{c}$ are transmitted, the echo signal data corresponding to each virtual element channel will transform to the following form after carrier frequency down-conversion, orthogonal demodulation, ${ }^{6}$ and range compression:

$$
s_{r c}\left(t, x_{m}, y_{n}\right)=\iiint_{\Omega} \operatorname{rect}\left[\frac{x_{m}}{L}\right] \operatorname{rect}\left[\frac{y_{n}-y_{p}}{L_{\mathrm{syn}}}\right] \sigma_{p} e^{-j \frac{4 \pi f c_{c}}{c} R^{\prime}} \operatorname{sinc}\left[B\left(t-\frac{2 R^{\prime}}{c}\right)\right] \mathrm{d} x_{p} \mathrm{~d} y_{p} \mathrm{~d} z_{p},
$$

where $t$ denotes the fast-time variable, $L_{\mathrm{syn}}$ denotes the along-track synthetic aperture length, and $c$ is the speed of light. For notational simplicity, we use rectangular window to represent the antenna patterns and signal weighing functions in Eq. (2). In some continuous wave radar, the wavenumber domain form of the range compressed signal $s_{r c}\left(t, x_{m}, y_{n}\right)$ is directly accessible, which is

$$
S_{r c}\left(k, x_{m}, y_{n}\right)=\iiint_{\Omega} \operatorname{rect}\left[\frac{k-k_{c}}{B}\right] \operatorname{rect}\left[\frac{x_{m}}{L}\right] \operatorname{rect}\left[\frac{y_{n}-y_{p}}{L_{\text {syn }}}\right] \times \sigma_{p} \exp \left(-j k 2 R^{\prime}\right) \mathrm{d} x_{p} \mathrm{~d} y_{p} \mathrm{~d} z_{p},
$$

where $k \in\left[k_{\min }, k_{\max }\right]$ is the fast-time wavenumber variable, with $k_{\min }=2 \pi\left(f_{c}-B / 2\right) / c$, $k_{\max }=2 \pi\left(f_{c}+B / 2\right) / c$, and $k_{c}=2 \pi f_{c} / c$.

Usually, the process of imaging is to estimate the distribution of the target reflectivity $\sigma_{p}$ from the range compressed signal $s_{r c}\left(t, x_{m}, y_{n}\right)$ or its wavenumber domain signal $S_{r c}\left(k, x_{m}, y_{n}\right)$. For example, in Ref. 9, after range compression, the compression in along-track is done using the synthetic aperture principle and the compression in cross-track is done using a digital beamforming. After that, true 3-D resolving ability is achieved so that the LA-3D-SAR can overcome the layover and shadowing effects in the conventional 2-D SARs. However, this is not the case when array errors, such as the multichannel amplitude-phase errors or virtual element position errors are present, where the signal coherency in the LA-3D-SAR is deteriorated and the final image may degrade drastically. Thus, it is essential to carry on a detailed investigation of the error effects and the related calibration methods.

\section{Array Errors and the Effects on LA-3D-SAR Imaging}

\subsection{Multichannel Amplitude-Phase Errors}

The multiple transmit and receive channels consist of active components (e.g., high frequency power amplifiers, mixers, intermediate frequency amplifiers and so on), whose amplitude and phase characteristics are slowly varying with the time. In addition, due to the nonideal electrical characteristic of antennas in the array, each channel may have a random deviation in the gain (or amplitude) and phase-shift from the ideal situation, which results in the multichannel amplitudephase errors. This type of error is independent of the along-track direction and mainly affects the imaging along the array (i.e., the cross-track direction), so that it is appropriate to simplify the analysis using only an equivalent 2-D geometric and signal model.

As shown in Fig. 2, $X$ and $Y^{\prime}$ represent the cross-track and slant range directions, respectively. Assume each virtual element's position is $\left(x_{m}, 0\right)$, where $m=0,1,2 \ldots M-1$. Using $R^{\prime}=\sqrt{y_{p}^{2}+\left(x_{m}-x_{p}\right)^{2}}$ to replace the instantaneous range in Eq. (1) and taking the 


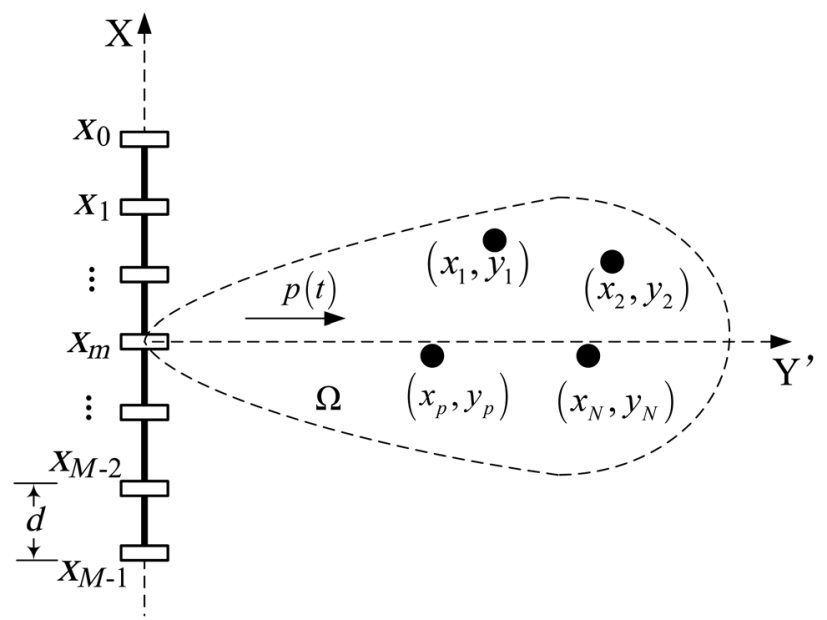

Fig. 2 Equivalent 2-D imaging geometry.

multichannel amplitude-phase error into consideration, the signal corresponding to the $m$ 'th channel can be expressed as

$$
\begin{aligned}
s_{m}(k) & =A_{m} \int_{\Omega} \sigma_{P} \times \exp \left\{-j k \cdot 2 \sqrt{y_{p}^{2}+\left(x_{m}-x_{p}\right)^{2}}+j \Delta \Phi_{m}\right\} \mathrm{d} \Omega \\
& =A_{m} \int_{\Omega} \sigma_{P} \times \exp \left\{-j k \cdot 2 \sqrt{y_{p}^{2}+\left(x_{m}-x_{p}\right)^{2}}+j\left(-k \cdot \Delta r_{m}+\phi_{m, c}\right)\right\} \mathrm{d} \Omega,
\end{aligned}
$$

where $A_{m}$ denotes the gain variations due to the amplitude error and $\Delta \Phi_{m}=-k \cdot \Delta r_{m}+\phi_{m, c}$ denotes the phase error, which can be divided into two parts: the first term $-k \cdot \Delta r_{m}$ representing the different time delay (or shift) components for each channel is referred to as range deviation phase error, and the other part $\phi_{m, c}$ is referred to as residue phase error.

For almost all the imaging algorithms, the cross-track focusing is done by coherent accumulation along the array direction, i.e.,

$$
s^{\prime}=\sum_{m=0}^{M-1} A_{m} e^{j \Phi} e^{j \Delta \Phi_{m}}=e^{j \Phi} \sum_{m=0}^{M-1} A_{m} e^{j \Delta \Phi_{m}},
$$

where $\Phi$ is the coherent phase that the algorithm compensates to. From Eq. (5), it is apparent that the target response does not achieve coherent accumulation because of the presence of multichannel amplitude-phase error $A_{m}$ and $\Delta \Phi_{m}$. According to the geometric and signal model, each antenna element in the array almost illuminates the same target area, so that it is reasonable to eliminate the target response deterioration introduced by the amplitude error $A_{m}$ by a simple power normalization of the channel data.

Compared to the amplitude error, the target response is more sensitive to the phase error. The range deviation phase error $-k \cdot \Delta r_{m}$ affects the imaging in a similar way as the range cell migration effects in conventional SAR imaging. We can use the integrated sidelobe ratio (ISLR) along the array as the figure of merit to evaluate the severity of the degradation induced by this error. Assume the channel number is $M=101$, center frequency $f_{c}=31$ $\mathrm{GHz}$, and the random range deviation corresponding to each channel conforms to the uniform distribution, i.e., $\Delta r_{m} \sim U[0, C], m=0,1,2 \ldots M-1$, where $C$ is a constant. Figure 3(a) shows the curve where the cross-track ISLR changes with parameter $C$. It is apparent that ISLR $=-10.43 \mathrm{~dB}$ for the error-free case, which accords with the ideal SAR impulse response, and the ISLR gets worse with the increase of range deviation error, e.g., when the deviation is beyond 0.3 wavelength, the target energy has spread out across the array totally, so that the ISLR measurement becomes meaningless.

The residue phase error $\phi_{m, c}$ in Eq. (4) manifests itself as a constant (within one channel) phase error attached to each different array channel. Let us denote the signal along the array 


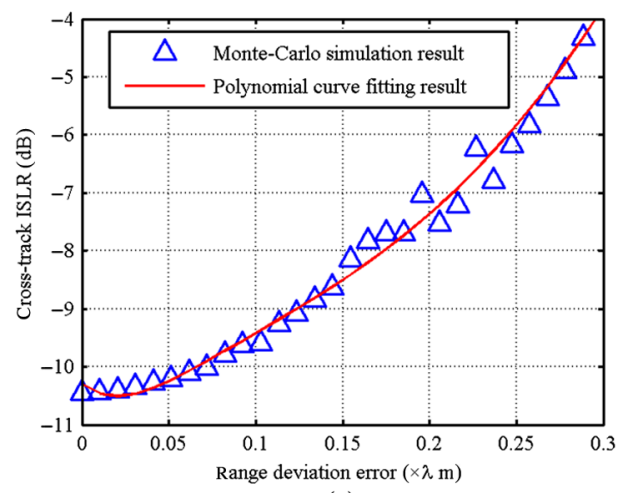

(a)

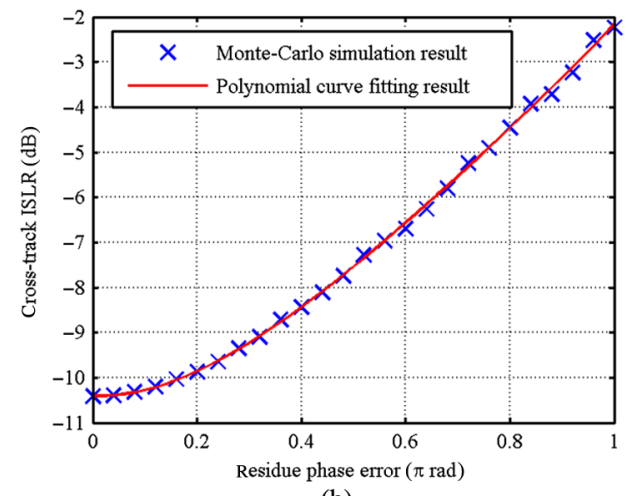

(b)

Fig. 3 Variation of cross-track ISLR with (a) the range deviation error and (b) the residue phase error.

direction as $s\left(x_{m}\right)$, and the ideal target response as $\operatorname{psf}_{\mathrm{x}}(x)$, then according to the Fourier transform equation, ${ }^{20}$ the practical signal $s\left(x_{m}\right) \cdot \exp \left\{j \phi_{m, c}\right\}$ can be processed after coherent accumulation, which yields

$$
s^{\prime}(x)=\operatorname{psf}_{\mathrm{x}}(x) * \operatorname{IDFT}\left\{\exp \left(j \phi_{m, c}\right)\right\},
$$

where $*$ denotes the discrete convolution. Equation (6) implies that the ideal target impulse response spreads over the $X$ axis because of the presence of $\operatorname{IDFT}\left\{\exp \left(j \phi_{m, c}\right)\right\}$. Again, using the ISLR evaluation methodology, except that the residue phase error $\phi_{m, c}$ is set to conform to a uniform distribution $\phi_{m, c} \sim U\left[0, C^{\prime} \cdot \pi\right], m=0,1,2 \ldots M-1$. As shown in Fig. 3(b), the target response is deteriorated with the increase of the residue phase error. For example, when $\phi_{m, c}$ distributes randomly between 0 and $\pi$, the level of ISLR has been creeping up from -3 to $-2 \mathrm{~dB}$, where correct imaging is impossible to achieve.

\subsection{Virtual Element Position Errors}

The virtual element position error is usually caused by wind deformation, gravity deformation, platform vibration, installation or motion measurement errors when a conformally designed antenna array is utilized in the airplane platform. It is usually expressed as the virtual element position deviation $\vec{\delta}_{m}=\Delta x_{m} \cdot \vec{i}_{x}+\Delta y_{m} \cdot \vec{i}_{y}+\Delta z_{m} \cdot \vec{i}_{z}$ from the ideal situation $\left(x_{m}, y_{n}, H\right)$, where $\vec{i}_{x}, \vec{i}_{y}$, and $\vec{i}_{z}$ represent the unit vector in cross-track, along-track, and height direction, respectively. An intuitive visualization of the virtual element position errors is provided in Fig. 4, where the actual position of the $m$ 'th virtual element is represented as $\left(x_{m}+\Delta x_{m}, y_{n}+\right.$ $\left.\Delta y_{m}, H+\Delta z_{m}\right)$, so the actual target-to-sensor distance is

$$
R^{\prime}=\sqrt{\left(x_{m}+\Delta x_{m}-x_{p}\right)^{2}+\left(y_{n}+\Delta y_{m}-y_{p}\right)^{2}+\left(H+\Delta z_{m}-z_{p}\right)^{2}} .
$$

From Eqs. (2) and (7), and using $r=(c / 2) t$, we can write the range compressed impulse response as

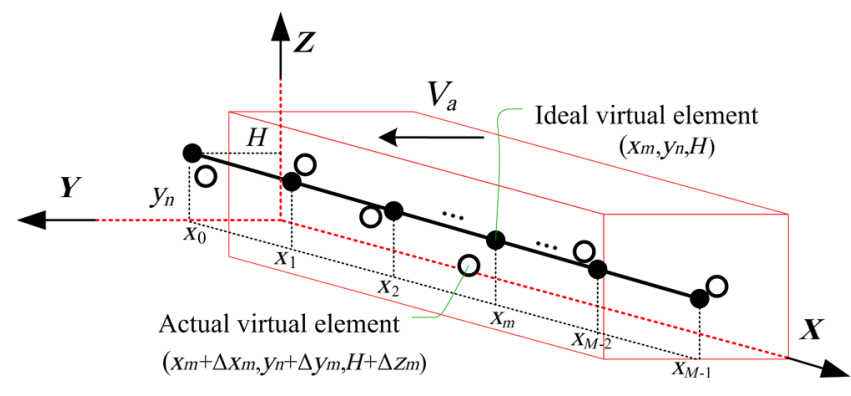

Fig. 4 Virtual element position errors. 


$$
s_{r}\left(r, x_{m}, y_{n}\right)=e^{-j k_{c} 2 R^{\prime}} \operatorname{psf}_{\mathrm{r}}\left(r-R^{\prime}\right)
$$

Taking the binomial expansion of Eq. (8), which yields

$$
\begin{aligned}
R^{\prime} \approx & R_{m 0}+\frac{\left(y_{n}-y_{p}\right)^{2}}{2 R_{m 0}}+\frac{\Delta x_{m}^{2}+\Delta y_{m}^{2}+\Delta z_{m}^{2}}{2 R_{m 0}} \\
& +\frac{\left(x_{m}-x_{p}\right) \Delta x_{m}}{R_{m 0}}+\frac{\left(y_{n}-y_{p}\right) \Delta y_{m}}{R_{m 0}}+\frac{\left(H-z_{p}\right) \Delta z_{m}}{R_{m 0}},
\end{aligned}
$$

where $R_{m 0}=\sqrt{\left(x_{m}-x_{p}\right)^{2}+\left(H-z_{p}\right)^{2}}$ is the minimum range between the point target to the $m$ 'th virtual element. The third term in Eq. (9) is so small that can be neglected, then inserting the remaining terms into Eq. (8), and neglecting the range curvature migration term in $\operatorname{psf}_{\mathrm{r}}(\cdot)$, results in

$$
s_{r}\left(r, x_{m}, y_{n}\right)=e^{-j k_{c} 2 R_{m 0}} e^{-j k_{c} \frac{\left(y_{n}-y_{p}\right)^{2}}{R_{m 0}}} e^{j\left(\Phi_{x}+\Phi_{y}+\Phi_{z}\right)} \operatorname{psf}_{\mathrm{r}}\left(r-R_{m 0}\right) .
$$

The first exponential term in Eq. (10) contains information for cross-track beamforming, the second exponential term is the modulation term for along-track compression, and $\Phi_{x}, \Phi_{y}$ and $\Phi_{z}$ represent phase error terms resulting from the virtual element position errors $\Delta x_{m}, \Delta y_{m}$, and $\Delta z_{m}$, respectively, which are defined as

$$
\left\{\begin{array}{l}
\Phi_{x}=-2 k_{c}\left(x_{m}-x_{p}\right) \Delta x_{m} / R_{m 0}, \\
\Phi_{y}=-2 k_{c}\left(y_{n}-y_{p}\right) \Delta y_{m} / R_{m 0}, \\
\Phi_{z}=-2 k_{c}\left(H-z_{p}\right) \Delta z_{m} / R_{m 0} .
\end{array}\right.
$$

As detailed in Ref. 5, for the range compressed data, a 3-D SAR image can be obtained by performing the along-track focusing and cross-track beamforming. However, the 3-D imaging quality is practically degraded by the phase error terms $\Phi_{x}, \Phi_{y}$, and $\Phi_{z}$ presented in Eq. (10) with high probability. In this paper, we relate the errors' effects to the image degradation by deriving appropriate statistical measures. We first model the virtual element position errors $\Delta x_{m}, \Delta y_{m}$, and $\Delta z_{m}$ as discrete, white, Gaussian stochastic process with zero-mean and variance of $\sigma_{x}^{2}, \sigma_{y}^{2}$, and $\sigma_{z}^{2}$, respectively. $\Delta x_{m}, \Delta y_{m}$, and $\Delta z_{m}$ are random variables over the cross-track virtual element positions and slowly time-varying along the along-track direction. From Eq. (11), we can see that $\Phi_{y}$ is a compound of linear phase error in the along-track direction and random phase error in the cross-track direction, whereas $\Phi_{x}$ and $\Phi_{z}$ are both random phase errors in the cross-track dimension.

In our previous research, ${ }^{21}$ we decompose the phase errors into different components and discuss their respective effects on imaging. Usually, a constant phase error component will have little effect on imaging, a linear phase error component will cause target displacement, a second-order component representing quadratic phase error will cause mainlobe broadening and sidelobe increase, and higher-order components manifesting as oscillation functions will cause paired echoes, which increase the integrated sidelobe level. We propose to use the discrete Legendre orthogonal transform ${ }^{22}$ to guarantee the mutual orthogonality of the expansion terms, and then appropriate statistical measures relating the effects of random phase errors to the degradation of the final image can be derived. From the analyses, it can be concluded that the virtual element position errors will contribute to a high cross-track integrated sidelobe level, which results in noise and contrast degradation in the image, and has little impact along the range and along-track directions.

Figure 5 shows the relationship between the error effects and the orthogonal expansion coefficients as well as the virtual element position error statistics for a typical Ka-band LA-3D-SAR parameters set. Let $a_{k}$ denote the $k^{\prime}$ th order orthogonal expansion term coefficient of $\Phi_{x}$, and $\sigma_{a_{k}}^{2}$ as its variance. Figure 5(d) implies that to meet a typical -17 dB ISLR demand for SAR imaging, ${ }^{23} \sigma_{a_{k}}^{2}$ has to be kept below $10^{-4}$. Otherwise, the resultant image will suffer from intolerable noise and contrast degradation in the image. Figure 5(e) shows the curves of the coefficient variance varying with $\sigma_{x}$, which are evaluated with four different point targets. For targets far away from the array, the coefficient variance is large and a $10^{-4}$ coefficient variance corresponds to $\sigma_{x}$ with the value of $0.5 \mathrm{~mm}$, which is taken as the calibration precision requirement 


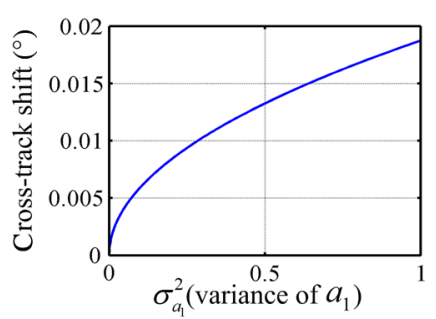

(a)

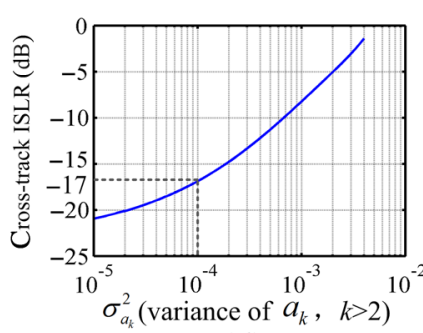

(d)

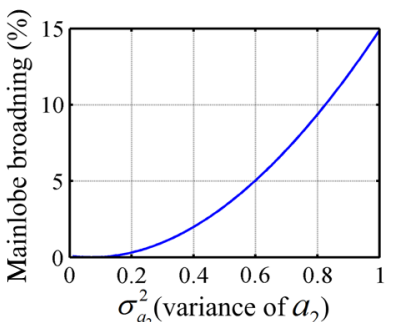

(b)

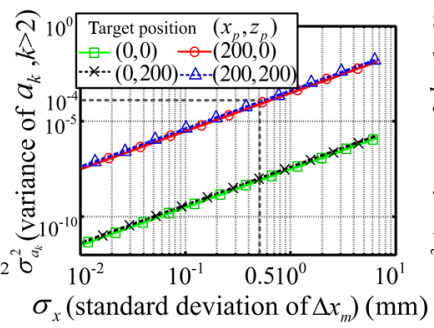

(e)

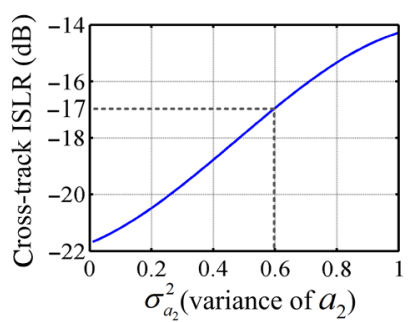

(c)

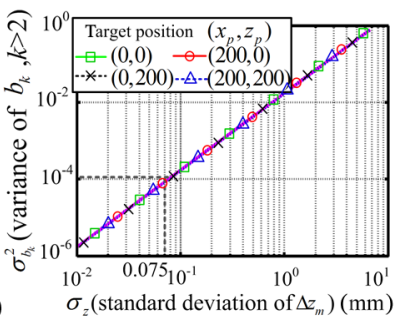

(f)

Fig. 5 Curves showing: (a) the cross-track displacement as a function of the first-order coefficient variance; (b) and (c) cross-track mainlobe broadening and ISLR as functions of the second-order coefficient variance; (d) cross-track ISLR as a function of the high-order coefficient variance; (e) and (f) the dependence of coefficient variance on the virtual element position errors. Ka-band is assumed where the parameters are the same as those given in Table 1 in Ref. 13.

of virtual element position error $\Delta x_{m}$. We can also derive from Figs. 5(a)-5(e) that, for virtual element position errors with millimeter-scale levels, the sidelobe increase due to paired echoes dominates the error effects, while the mainlobe is almost intact due to small low-order phase error components. Figure 5(f) gives the curves of the coefficient variance $\sigma_{b_{k}}^{2}$ of $\Phi_{z}$ changing with the standard deviation $\sigma_{z}$ of the virtual element position error component $\Delta z_{m}$, again evaluated with four point targets located at different positions. It is apparent that the four curves almost overlapped, and a $10^{-4}$ coefficient variance corresponds to $\sigma_{z}$ value of approximately $0.075 \mathrm{~mm}$. Some calibration or compensation procedures would be necessary if $\Delta z_{m}$ exceeds this limit.

Compared with $\Delta y_{m}$, the beamforming performance along the cross-track is more sensitive to $\Delta z_{m}$, which is illustrated in Fig. 6 with a Ka-band point analysis example. In Fig. 6(a), though the mainlobe of the cross-track impulse response is intact as the error-free case, the impulse response is corrupted by high sidelobes (an ISLR above $0 \mathrm{~dB}$ ) resulting from virtual element position errors when $\sigma_{x}=5 \mathrm{~mm}$. When $\sigma_{x}$ is reduced to $0.5 \mathrm{~mm}$, as shown in Fig. 6(b), the ISLR is reduced to an acceptable level of $-17 \mathrm{~dB}$ correspondingly, which satisfies the imaging requirement. However, as shown in Fig. 6(c), the same $0.5 \mathrm{~mm}$ level of errors as applied in the $Z$ direction will still spread out energy across the impulse response and give rise to an ISLR of $-1.5 \mathrm{~dB}$. In Fig. 6(d), when the $\sigma_{z}$ is set to $0.075 \mathrm{~mm}$, a favorable cross-track impulse response with ISLR of $-17 \mathrm{~dB}$ is obtained. Thus, in order to mitigate the impact of the rising sidelobes, calibration measures should be taken to keep the error below a tolerable level, and the precision requirement of $\Delta z_{m}$ should be taken as a reference for calibrating the virtual element position errors.

\section{Calibration of the Array Errors}

\subsection{Calibration of the Multichannel Amplitude-Phase Errors}

\subsubsection{Amplitude error calibration using power normalization}

For LA-3D-SAR imaging, each antenna element in the array almost illuminates the same target area, which implies that the amplitude variations among different channel data are caused by the random amplitude error $A_{m}$, thus can be compensated by a simple power (sum of the magnitude 


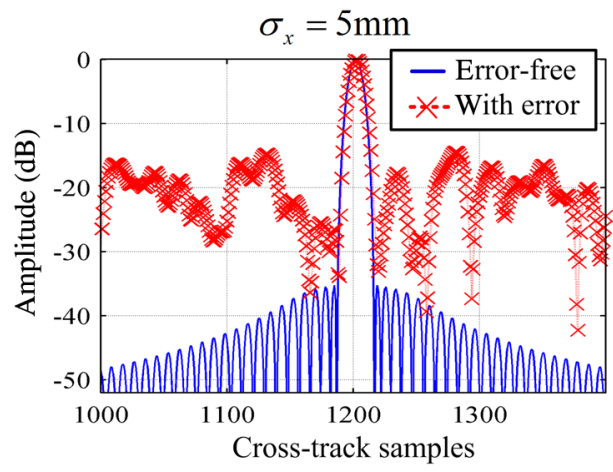

(a)

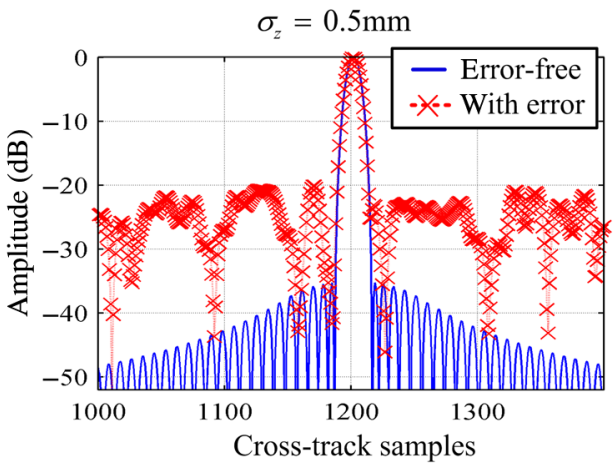

(c)

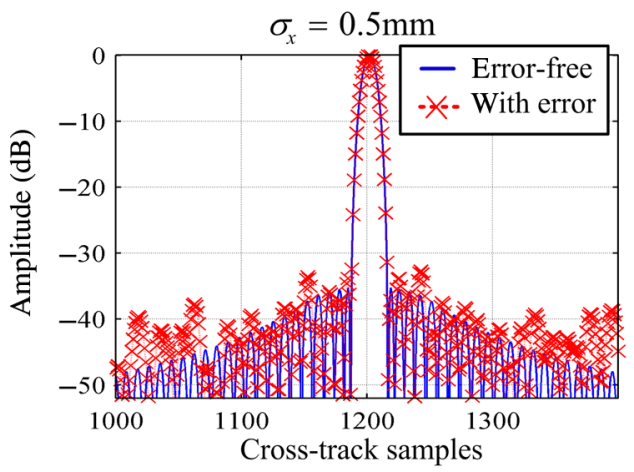

(b)

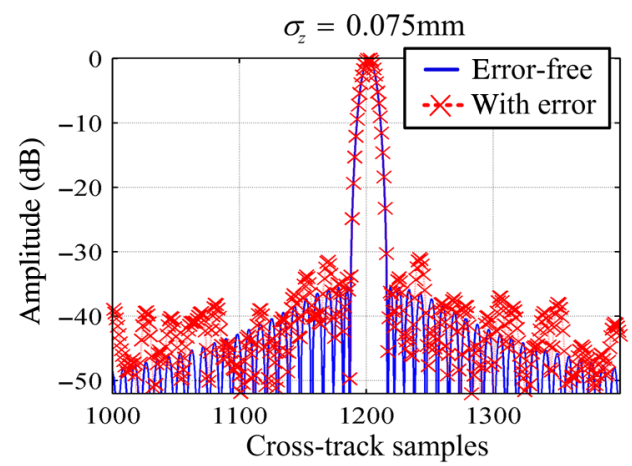

(d)

Fig. 6 The cross-track impulse response when the virtual element position errors $\Delta y_{n}$ or $\Delta z_{n}$ exists.

squared) normalization of the channel data, i.e., assuming the signal corresponding to the $m$ 'th channel is $s_{m}(n)$, with the power value of $E_{m}$ and fast-time samples number $N$, then power normalization can be done through

$$
\tilde{s}_{m}(n)=\frac{s_{m}(n)}{\sqrt{E_{m}}}, \quad m=0,1, \cdots, M-1, \quad n=1,2, \cdots, N,
$$

where $\tilde{s}_{m}(n)$ is the $m$ 'th channel data after amplitude error calibration.

\subsubsection{Phase error calibration using external calibrators}

The multichannel phase errors can be calibrated on a static platform indoors before the array is applied on the airplane platform. Due to the slowly-varying characteristic of the phase errors, the calibration parameters can be stored onboard for the SAR processor to look up and compensate within a certain period of time.

Figure 7 shows the calibration geometries using two different external calibrators. Figure 7(a) uses an external calibrator, which is placed in parallel to the array direction, such that the distance (range) histories and phase histories are expected to be the same for all channel data. Figure 7(b) uses a point target (e.g., a corner reflector) with strong reflectivity as the external calibrator, whereby the phase error calibration parameters can be estimated by virtue of the triangular geometry relationship of the array and the point target calibrator.

More specifically, take the calibration procedure in Fig. 7(a) as an example. First, the range compressed data (in 2-D time or spatial domain) are upsampled along the fast-time direction, then the distance histories $\hat{R}_{m}$ of the calibrator in all channels are estimated by cross-correlating the data with the range point spread function $\operatorname{psf}_{\mathrm{r}}(t)$ :

$$
\left\{\begin{array}{c}
\mu_{m}(\tau)=\int_{-\infty}^{\infty} s_{m}(t) \operatorname{psf}_{\mathrm{r}}(t-\tau) \mathrm{d} t \\
\hat{\tau}_{m}=\arg \max _{\tau} \mu_{m}(\tau) \\
\hat{R}_{m}=\frac{c}{2} \times \hat{\tau}_{m} .
\end{array}\right.
$$




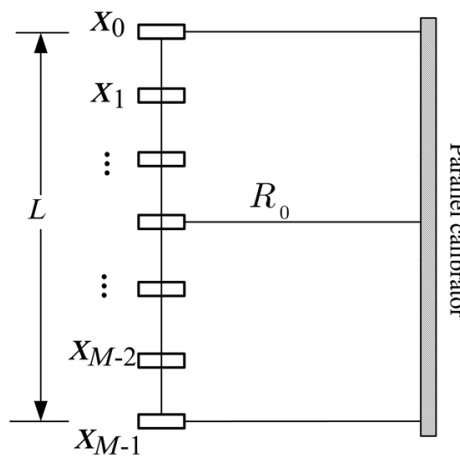

(a)

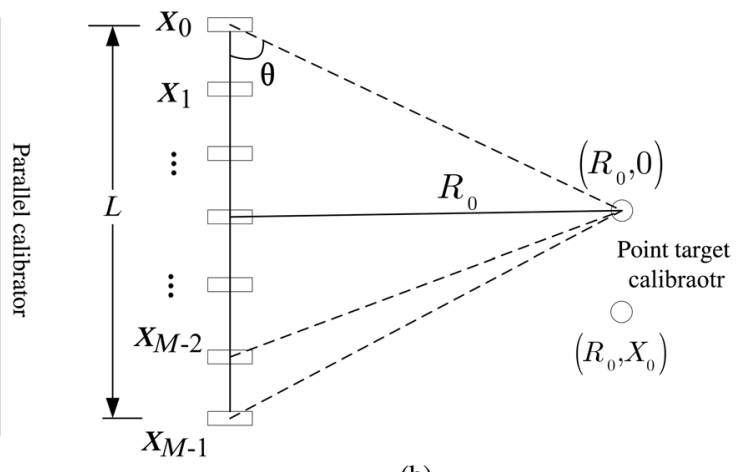

(b)

Fig. 7 Calibration geometry: (a) with a parallel external calibrator and (b) with a point target calibrator.

Then the difference of $\hat{R}_{m}$ and the ideal distance value $R_{0}$, i.e., $\Delta \hat{r}_{m}=\hat{R}_{m}-R_{0}$, is taken as the estimated value of the range deviation error corresponding to the $m$ 'th channel:

$$
S_{m}^{\prime}(k)=S_{m}(k) \times \exp \left\{j 2 k \Delta \hat{r}_{m}\right\}
$$

After correcting the range deviations, the energy trajectory of the calibrator locates at the range gate of $R_{0}$ and manifests as a parallel line with respect to the array. Then the phase values $\hat{\phi}_{m, c}$, $m=0,1, \ldots, M-1$ on the calibrator response peak can be extracted as the estimated residue phase errors, and thus can be corrected through

$$
S_{m}^{\prime \prime}(k)=S_{m}^{\prime}(k) \times \exp \left\{-j \hat{\phi}_{m c}\right\} .
$$

The calibration method in Fig. 7(b) is similar, except that the ideal energy trajectory exhibits the form of a hyperbolic curve rather than a straight line, and the range deviation phase error values are estimated by virtue of the triangular geometry, whereas the residue phase error values are extracted along the curved energy trajectory. Ground-based experiment examples given in the latter part of the paper have confirmed the feasibility of the calibration method.

\subsection{Calibration of the Virtual Element Position Errors Using Time-Divided Active Calibrators}

For the virtual element position errors, one way of calibration is to utilize distributed motion measurement units to retrieve the exact position of each antenna and then correct the error effects by postprocessing. ${ }^{24}$ However, considering the big amount of elements and the limited precision of the state-of-the-art measurement device, this approach will be too complicated and expensive.

Another feasible approach is to use the array calibration methods in array signal processing theory. ${ }^{25-27}$ In the downward-looking LA-3D-SAR imaging model, $\Delta x_{m}, \Delta y_{m}$, and $\Delta z_{m}$ are random variables over the cross-track virtual element positions and slowly time-varying over the along-track integration time, which means that for a set of adjacent along-track spatial samples $y_{n}$, the position errors are deterministic and constant. For a certain $y_{n}$, the cross-track beamforming is analogous to the direction of arrival (DOA) estimation problem ${ }^{28}$ in array signal processing theory.

In a typical DOA radar, the array calibration methods usually use active calibrators (or signal sources) that have a priori position and angle (DOA) information relative to the array. By reversely deriving the DOA estimation algorithm (e.g., the MUSIC algorithm ${ }^{29}$ ), the actual antenna phase centers of the array elements can be retrieved. For a fixed array, the steering vector is determined by the element positions and the calibrator's DOA, so that the element position errors are also involved in the steering vector, which can be retrieved by some optimization methods. The virtual element position errors in LA-3D-SAR can be calibrated in a similar manner, except that the data channels are composed of virtual elements formed by aperture synthesis of a sparse MIMO array, rather than of real receiving elements. 
Tan et al.: Array error calibration methods in downward-looking linear-array...

A practical solution is to use active calibrators that have relay functions, such as active transponders. ${ }^{30}$ Such a device is analogous to a corner reflector, in which the calibrator's receiving antenna receives the SAR signal and then feeds it back to the radar sensor after gain amplification. The transponders adopt transparent relay technology such that the signal phase is preserved and the signal characteristics are not deteriorated. The calibrators can be arranged in the illuminated scene, where the angle information (DOA) with respect to the array in the airborne platform can be accurately measured by ground and airborne position measurement devices. Using this information, the virtual element position errors can be calibrated by estimating the steering vectors of the array.

The following is a detailed description of our proposed calibration method, which is based on time-divided active calibrators. Here, "time-divided" means that the active calibrators are activated time-dividedly one after another, at different along-track times during the calibration process. As shown in Fig. 8, set the central virtual element of the cross-track array as the reference and assuming the along-track time $\eta=0$, so that the reference element is located at the coordinate origin $(0$, $0,0)$ and is assumed to be error-free. Assume a calibrator (transponder) $P_{i}$ is located at $\left(x_{i}, y_{i}, z_{i}\right)$ in Fig. 8, where $\alpha_{i}$ and $\beta_{i}$ represent the along-track and cross-track looking angle with respect to the cross-track array. At time $\eta$, let $y_{n}$ denote the along-track position of the LA-3D-SAR, the $M_{\mathrm{T}}$ transmitting antennas emit orthogonal waveforms $p_{0}(t), p_{1}(t), \ldots, p_{M_{\mathrm{T}}-1}(t)$, the calibrator $P_{i}$ relays the signals and later, the signals are received by all $M_{\mathrm{R}}$ receiving antennas simultaneously. By aperture synthesis, a total number of $M$ virtual elements are formed and the channel data corresponding to the $m$ 'th virtual element are

$$
s_{m}(r)=e^{-j k_{c} 2 R^{\prime}} \operatorname{psf}_{\mathrm{r}}\left(r-R^{\prime}\right)+N_{m}(r),
$$

where $r$ is the fast-time range variable, $k_{c}$ is the center wave number, $N_{m}(r)$ represents additive channel noise which is a Gaussian, white stochastic process and independent of the signal components, $R^{\prime}$ is the instantaneous range from the $m^{\prime}$ th virtual element to the calibrator, with the expression:

$$
R^{\prime}=\sqrt{\left(x_{m}+\Delta x_{m}-x_{i}\right)^{2}+\left(y_{n}+\Delta y_{m}-y_{i}\right)^{2}+\left(H+\Delta z_{m}-z_{i}\right)^{2}} .
$$

Let $R_{i 0}=\sqrt{\left(y_{n}-y_{i}\right)^{2}+x_{i}^{2}+\left(H-z_{i}\right)^{2}}$ denote the range from the calibrator to the reference element, then taking the Taylor series expansion of $R^{\prime},{ }^{31}$ and neglecting the small quadratic and higher-order terms, yields

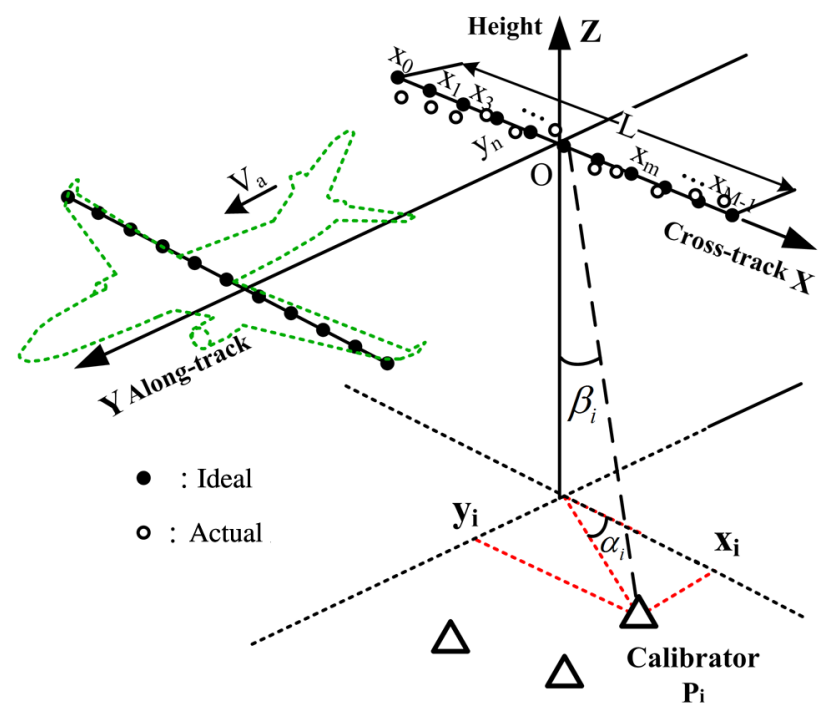

Fig. 8 Calibration of virtual element position error using time-divided active calibrators. 
Tan et al.: Array error calibration methods in downward-looking linear-array...

$$
\begin{aligned}
R^{\prime} \approx & \sqrt{R_{i 0}^{2}-2\left(y_{n}-y_{i}\right) \Delta y_{m}-2\left(x_{m}+\Delta x_{m}\right) x_{i}+2\left(H-z_{i}\right) \Delta z_{m}} \\
\approx & R_{i 0}-\frac{\left(y_{n}-y_{i}\right)}{\sqrt{\left(y_{n}-y_{i}\right)^{2}+x_{i}^{2}}} \frac{\sqrt{\left(y_{n}-y_{i}\right)^{2}+x_{i}^{2}}}{R_{i 0}} \Delta y_{m} \\
& -\frac{x_{i}}{\sqrt{\left(y_{n}-y_{i}\right)^{2}+x_{i}^{2}}} \frac{\sqrt{\left(y_{n}-y_{i}\right)^{2}+x_{i}^{2}}}{R_{i 0}}\left(x_{m}+\Delta x_{m}\right)+\frac{\left(H-z_{i}\right)}{R_{i 0}} \Delta z_{m} \\
= & R_{i 0}-\Delta y_{m} \sin \left(\alpha_{i}\right) \sin \left(\beta_{i}\right)-\left(x_{m}+\Delta x_{m}\right) \cos \left(\alpha_{i}\right) \sin \left(\beta_{i}\right)+\Delta z_{m} \cos \left(\beta_{i}\right) .
\end{aligned}
$$

Inserting Eq. (18) into Eq. (16), and transforming the result into vector-matrix form, which yields

$$
\mathbf{r}=\tilde{\mathbf{a}}(\alpha, \beta) s(t)+\mathbf{N}
$$

where

$$
\begin{aligned}
& \mathbf{r}=\left[\begin{array}{llll}
s_{0}(r) & s_{1}(r) & \cdots & s_{M-1}(r)
\end{array}\right]^{T}, \\
& \mathbf{a}\left(\alpha_{i}, \beta_{i}\right)=\left[\exp \left\{j 2 k_{c} x_{0} \cos \left(\alpha_{i}\right) \sin \left(\beta_{i}\right)\right\}, \ldots, \exp \left\{j 2 k_{c} x_{M-1} \cos \left(\alpha_{i}\right) \sin \left(\beta_{i}\right)\right\}\right]^{T}, \\
& \tilde{\mathbf{a}}\left(\alpha_{i}, \beta_{i}\right)=\Delta_{i} \mathbf{a}\left(\alpha_{i}, \beta_{i}\right), \\
& \Delta_{i}=\operatorname{diag}\left\{\left[\exp \left\{j \varphi_{0}\left(\alpha_{i}, \beta_{i}\right)\right\} \quad \cdots \quad \exp \left\{j \varphi_{M-1}\left(\alpha_{i}, \beta_{i}\right)\right\}\right]^{T}\right\}, \\
& \varphi_{m}\left(\alpha_{i}, \beta_{i}\right)=2 k_{c}\left[\Delta y_{m} \sin \left(\alpha_{i}\right) \sin \left(\beta_{i}\right)+\Delta x_{m} \cos \left(\alpha_{i}\right) \sin \left(\beta_{i}\right)-\Delta z_{m} \cos \left(\beta_{i}\right)\right], \\
& s(r)=e^{-j k_{c} 2 R_{0 i}} \operatorname{psf}_{\mathrm{r}}\left(r-R^{\prime}\right), \\
& \mathbf{N}=\left[\begin{array}{llll}
N_{0}(t) & N_{1}(t) & \cdots & N_{M-1}(t)
\end{array}\right]^{T},
\end{aligned}
$$

where $[\cdot]^{T}$ represents vector transpose, $\operatorname{diag}\{\mathbf{v}\}$ represents a diagonal matrix whose diagonal is vector $\mathbf{v}$, Eq. (21) is the error-free steering vector of the array, whereas Eq. (22) is the steering vector when virtual element position errors exist.

The covariance matrix of the array signal $\mathbf{r}$ is

$$
\mathbf{R}_{r r}=E\left[\mathbf{r r}^{H}\right]=\tilde{\mathbf{a}} E\left[\mathbf{S S}^{H}\right] \tilde{\mathbf{a}}^{H}+\sigma^{2} \mathbf{I}=\tilde{\mathbf{a}} \mathbf{R}_{S} \tilde{\mathbf{a}}^{H}+\sigma^{2} \mathbf{I},
$$

where $[.]^{H}$ represents Hermitian transpose. Because the signal and noise are independent, the covariance matrix can be decomposed into two uncorrelated parts corresponding to the signal and noise. Let us take the eigenvalue decomposition of matrix $\mathbf{R}_{r r}$ :

$$
\mathbf{R}_{r r}=\mathbf{U}_{\mathbf{S}} \sum_{\mathbf{S}} \mathbf{U}_{\mathbf{S}}^{\mathbf{H}}+\mathbf{U}_{\mathbf{N}} \sum_{\mathbf{N}} \mathbf{U}_{\mathbf{N}}^{\mathbf{H}}
$$

In Eq. (28), $\mathbf{U}_{\mathbf{S}}$ represents the signal subspace spanned by the eigenvectors corresponding to big eigenvalues, whereas $\mathbf{U}_{\mathbf{N}}$ represents the noise subspace spanned by the eigenvectors corresponding to small eigenvalues. Assuming that there is only one active calibrator in the scene, according to the MUSIC algorithm, the eigenvector of the covariance matrix of the array output corresponding to the largest eigenvalue is proportional to the steering vector. Let $\mathbf{q}$ denote this eigenvector, then

$$
\hat{\tilde{\mathbf{a}}}\left(\alpha_{i}, \beta_{i}\right)=c_{0} \mathbf{q}
$$

where $c_{0}$ is a real constant. $\hat{\tilde{\mathbf{a}}}\left(\alpha_{i}, \beta_{i}\right)$ is the estimated steering vector that contains virtual element position errors. From Eq. (22)-(24), we can estimate the diagonal matrix $\Delta_{i}$ by solving the following optimization problem: 


$$
\hat{\Delta}_{i}=\arg \min _{\Delta_{i}}\left[\hat{\tilde{\mathbf{a}}}\left(\alpha_{i}, \beta_{i}\right)-\Delta_{i} \mathbf{a}\left(\alpha_{i}, \beta_{i}\right)\right]^{\mathrm{H}}\left[\hat{\mathbf{a}}\left(\alpha_{i}, \beta_{i}\right)-\Delta_{i} \mathbf{a}\left(\alpha_{i}, \beta_{i}\right)\right]
$$

s.t.the matrix $\Delta_{i}$ with M rows and M columns is diagonal.

Let $b_{m}$ and $a_{m}$ denote the $m^{\prime}$ th elements of vectors $\hat{\tilde{\mathbf{a}}}\left(\alpha_{i}, \beta_{i}\right)$ and $\mathbf{a}\left(\alpha_{i}, \beta_{i}\right)$, respectively, i.e., $\hat{\tilde{\mathbf{a}}}\left(\alpha_{i}, \beta_{i}\right)=\left[b_{1}, \cdots b_{m}, \cdots b_{M}\right]^{T}$ and $\mathbf{a}\left(\alpha_{i}, \beta_{i}\right)=\left[a_{1}, \cdots a_{m}, \cdots a_{M}\right]^{T}$, and considering that $\Delta_{i}$ is a diagonal square matrix, i.e., $\Delta_{i}=\operatorname{diag}\left(x_{1}, \cdots x_{m}, \cdots x_{M}\right)$, then it is easy to get

$$
\begin{aligned}
& {\left[\hat{\tilde{\mathbf{a}}}\left(\alpha_{i}, \beta_{i}\right)-\Delta_{i} \mathbf{a}\left(\alpha_{i}, \beta_{i}\right)\right]^{\mathrm{H}}\left[\hat{\tilde{\mathbf{a}}}\left(\alpha_{i}, \beta_{i}\right)-\Delta_{i} \mathbf{a}\left(\alpha_{i}, \beta_{i}\right)\right]} \\
& \quad=\left|b_{1}-x_{1} a_{1}\right|^{2}+\ldots+\left|b_{m}-x_{m} a_{m}\right|^{2}+\ldots+\left|b_{M}-x_{M} a_{M}\right|^{2} \geq 0 .
\end{aligned}
$$

Because the above expression is non-negative, the minimization is achieved when each additional term of $\left|b_{m}-x_{m} a_{m}\right|^{2}$ in Eq. (31) becomes 0, which means that the estimated value of $x_{m}$ is $b_{m} / a_{m}$, so that

$$
\hat{\Delta}_{i}=\operatorname{diag}\left(\frac{b_{1}}{a_{1}}, \cdots, \frac{b_{m}}{a_{m}}, \cdots, \frac{b_{M}}{a_{M}}\right)
$$

for which $a_{m}, m=1,2, \ldots M$ all have nonzero values according to the definition of $\mathbf{a}\left(\alpha_{i}, \beta_{i}\right)$ in Eq. (21).

Suppose there are a total number of $K$ time-divided active calibrators in the scene. For each calibrator, we can obtain an estimated diagonal matrix $\hat{\Delta}_{i}$, let $\mathbf{g}_{i}$ denote the vector formed by the diagonal elements of matrix $\hat{\Delta}_{i}$, then the virtual antenna element position errors can be estimated by solving the matrix equation:

$$
\mathbf{g}=\mathbf{T} \delta
$$

where

$$
\begin{aligned}
& \mathbf{g}=\left[\begin{array}{llll}
\operatorname{Arg}\left\{\mathbf{g}_{1}\right\} & \operatorname{Arg}\left\{\mathbf{g}_{2}\right\} & \cdots & \operatorname{Arg}\left\{\mathbf{g}_{K}\right\}
\end{array}\right]_{K \times M}^{T}, \\
& \delta=\left[\begin{array}{llll}
\Delta x_{0} & \Delta x_{1} & \cdots & \Delta x_{M-1} \\
\Delta y_{0} & \Delta y_{1} & \cdots & \Delta y_{M-1} \\
\Delta z_{0} & \Delta z_{1} & \cdots & \Delta z_{M-1}
\end{array}\right]_{3 \times M} \\
& \mathbf{T}=2 k_{c}\left[\begin{array}{ccc}
\sin \left(\alpha_{1}\right) \sin \left(\beta_{1}\right) & \cos \left(\alpha_{1}\right) \sin \left(\beta_{1}\right) & -\cos \left(\beta_{1}\right) \\
\sin \left(\alpha_{2}\right) \sin \left(\beta_{2}\right) & \cos \left(\alpha_{2}\right) \sin \left(\beta_{2}\right) & -\cos \left(\beta_{2}\right) \\
\vdots & \vdots & \vdots \\
\sin \left(\alpha_{K}\right) \sin \left(\beta_{K}\right) & \cos \left(\alpha_{K}\right) \sin \left(\beta_{K}\right) & -\cos \left(\beta_{K}\right)
\end{array}\right]_{K \times 3}
\end{aligned}
$$

where $\operatorname{Arg}\{\cdot\}$ is the operator for obtaining the angle of a complex quantity. Because $\delta$ contains $3(M-1)$ unknown variables and each calibrator can provide $(M-1)$ equations to the linear system, then there should exist at least three calibrators to solve the matrix equation. If $K=3$, the unknown variables can be determined uniquely by multiplying the inverse of a nonsingular matrix:

$$
\delta=\mathbf{T}^{-1} \mathbf{g}
$$

In such a case, the DOA angular interval among the calibrators should not be too close in order to avoid the matrix singularity. If more than three calibrators are provided, i.e., $K>3$, we say the linear system is overdetermined and thus can be solved by the least square method. ${ }^{32}$ However, if $K<3$, then the linear system is underdetermined, which means the unknowns cannot be determined uniquely. So, to complete the calibration mission in practice, more than three calibrators with a dispersed distribution in the scene are preferable. 


\section{Experimental Verification Results}

\subsection{Indoor Experiments of the Multichannel Amplitude-Phase Error Calibration}

\subsubsection{Two-dimensional imaging experiment}

To verify the multichannel amplitude-phase error calibration methods, we utilize a Ka-band horn antenna array horizontally placed on a static indoor platform, which is shown in Fig. 9. An Agilent E8363C vector network analyzer (VNA) is used as the signal transceiver. ${ }^{33}$ With the help of a microwave multiplexer to switch the transmit and receive channel from one antenna to another on a pulse to pulse basis, a linear array with 60 virtual elements and an 8-mm innerelement spacing is synthesized. The test target scenario is composed of three metallic spheres, and before acquiring the backscattered data from them, a metal sheet functioning as a calibrator is placed parallel to the array. The experiment parameters are listed in Table 1.

The calibration procedure is implemented following Eqs. (12)-(15), then the calibration parameters are applied, and the results are shown in Fig. 10. Figure 10(b) shows the degraded imaging result without the multichannel amplitude-phase error correction. It is apparent that the image is defocused with blurred and ghost targets, which are caused by channel inconsistency. After the calibration parameters are applied, a clear focused image shown in Fig. 10(c) was obtained, within which the geometric locations of all three target spheres accord with those of the true scenario, as shown in the photo of Fig. 10(a).

\subsubsection{Three-dimensional imaging experiment}

A 3-D imaging experiment with the same Ka-band array as in Fig. 9 is also conducted to verify the calibration method. As seen in Fig. 11, the array is vertically fixed on a horizontal rail of a ground-based platform. ${ }^{34}$ When the VNA is programmed to transmit and receive signal pulses, a synthetic aperture is realized by scanning the array along the horizontal rail and a virtual array aperture is realized by aperture synthesis of the antennas. The geometry assembles a downwardlooking LA-3D-SAR in that it is simply a rotation version of the coordinates introduced in Fig. 1. In the current experiment, the array antennas synthesize a linear array with 65 virtual elements

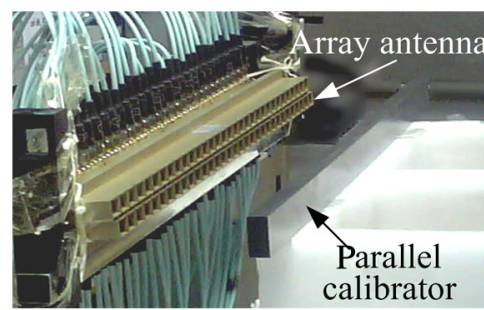

(a)

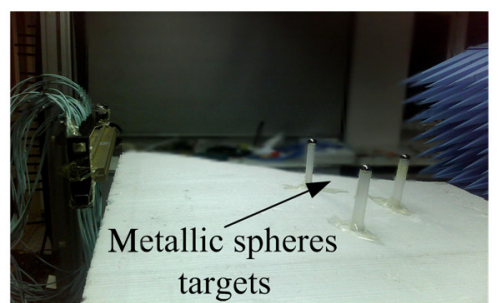

(b)

Fig. 9 2-D imaging experiment setup for calibrating the multichannel amplitude-phase errors.

Table $12-\mathrm{D}$ imaging experiment parameters.

\begin{tabular}{lr}
\hline \hline Parameters & Value \\
\hline Center frequency & $31 \mathrm{GHz}$ \\
Frequency bandwidth & $6 \mathrm{GHz}$ \\
Frequency points & 1601 \\
Array aperture length & $0.472 \mathrm{~m}$ \\
Inner-element spacing & $8 \mathrm{~mm}$ \\
Virtual element number & 60 \\
\hline \hline
\end{tabular}




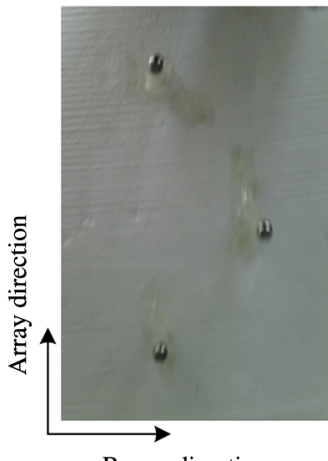

Range direction

(a)

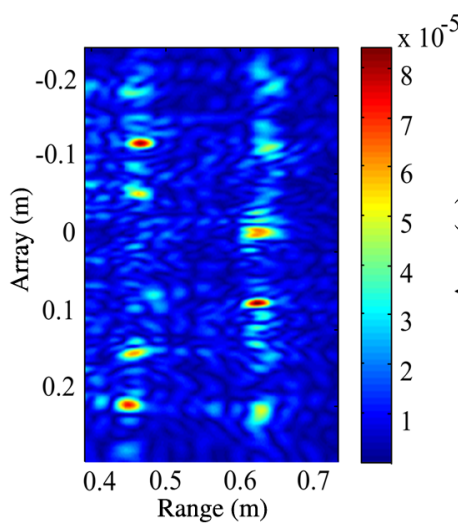

(b)

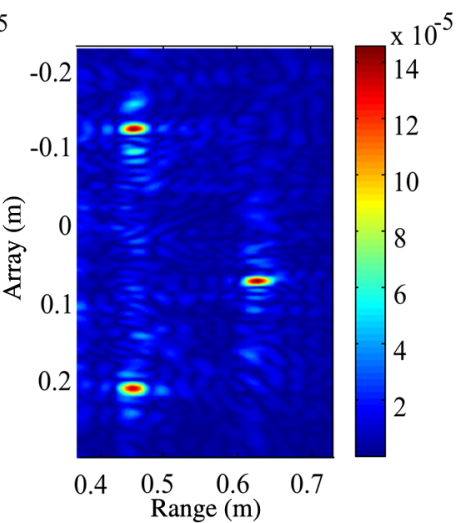

(c)

Fig. 10 2-D imaging experiment: (a) target scene with three metallic spheres; (b) 2-D imaging result without any calibration; and (c) 2-D imaging result after calibration.
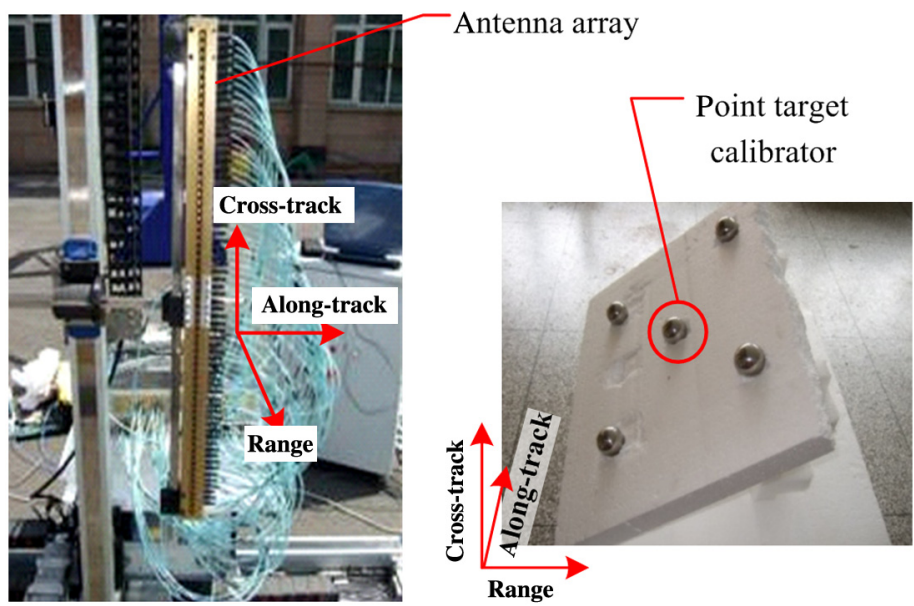

Fig. 11 3-D imaging experiment setup for calibrating the multichannel amplitude-phase errors.

and an 8-mm inner-element spacing. The target scenario comprises five metallic spheres in front of the array and the experiment parameters are listed in Table 2.

The phase error is calibrated by setting one point scatterer (the central metallic sphere in this experiment) as the reference target and calculating the difference between the ideal phase history along the antenna array and the real phase extracted from the 2-D compressed image

Table 2 3-D imaging experiment parameters.

\begin{tabular}{lr}
\hline \hline Parameters & Value \\
\hline Center frequency & $35 \mathrm{GHz}$ \\
Frequency bandwidth & $4 \mathrm{GHz}$ \\
Frequency points & 801 \\
Inner-element spacing & $8 \mathrm{~mm}$ \\
Virtual element number & 65 \\
Movement interval on rail & $8 \mathrm{~mm}$ \\
Samples number on rail & 101 \\
\hline \hline
\end{tabular}




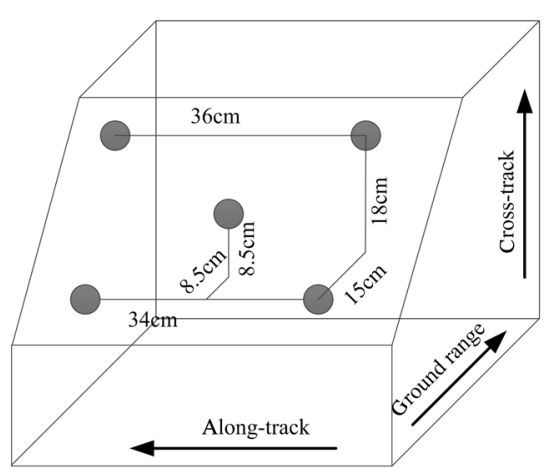

(a)

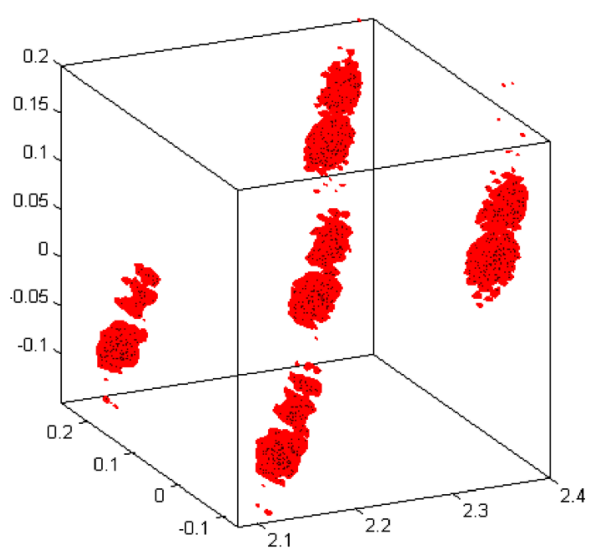

(c)

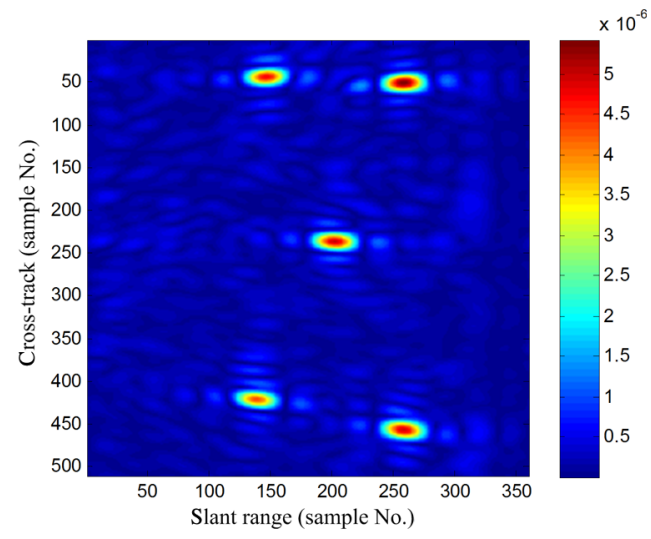

(b)

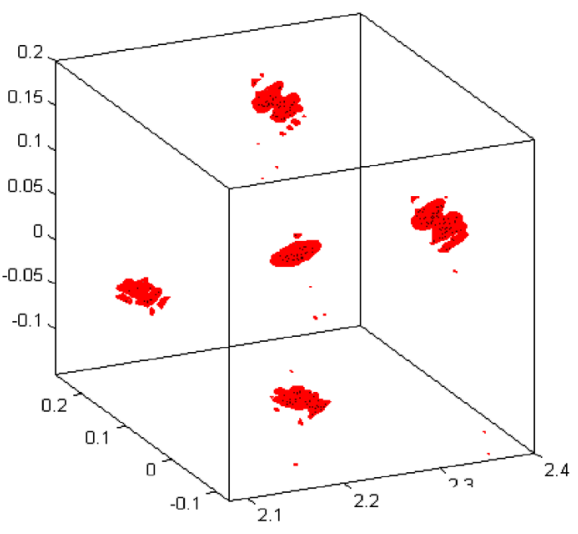

(d)

Fig. 12 3-D imaging experiment: (a) target scene set up with five metallic spheres; (b) 2-D imaging result corresponding to the 40th data channel; (c) 3D imaging result without any calibration; and (d) 3-D imaging result after calibration.

corresponding to each channel. The ideal phase is obtained with the help of the relative geometric relationship between the array and the reference target and the extraction of the real phase can be performed on the peak location in the 2-D image. Then the phase error can be compensated and hence, an exact 3-D image could be expected.

In Fig. 12, we show the reconstructed 3-D images of the measurement data with and without the calibration steps, thus the importance of amplitude-phase error compensation is demonstrated. In Fig. 12(b), a 2-D image corresponding to the 40th data channel was displayed with which we can clearly see focused five spheres. The 2-D images of the other channels are similar. Figure 12(c) shows the defocused 3-D image obtained without any error compensation, whereas Fig. 12(d) gives the 3-D result after calibration. In comparison with Fig. 12(c), the five targets in Fig. 12(d) are focused in all three dimensions with higher precision. And the locations of the focused targets are in exact accordance with the target scene setup in Fig. 12(a). However, a slight defocusing still exists and this may result from the slight vibration of the array during scanning along the horizontal rail.

\subsection{Numerical Simulations of the Virtual Element Position Error Calibration}

We test the calibration method with a simulated 3-D distributed target scene with the typical Kaband LA-3D-SAR parameters listed in Table 3. The target scene comprises scatterers, whose locations and reflectivities come from a 2-D CSAR image and its digital elevation model (DEM) data, ${ }^{35}$ as shown in Fig. 13. The virtual element position errors are artificially added to the SAR raw data, where $\sigma_{x}, \sigma_{y}$, and $\sigma_{z}$ are both set to $1 \mathrm{~mm}$. Three time-divided active calibrators are used and the SNR is set to be $20 \mathrm{~dB}$. 
Tan et al.: Array error calibration methods in downward-looking linear-array...

Table 3 Typical Ka-band parameters for simulation use. ${ }^{a}$

\begin{tabular}{lccc}
\hline \hline Parameters & Value & Parameters & Value \\
\hline Airplane height $H$ & $1500 \mathrm{~m}$ & Center frequency $f_{c}$ & $36.5 \mathrm{GHz}$ \\
Array length $L$ & $4 \mathrm{~m}$ & Element number $N$ & 268 \\
$\Delta x_{n}, \Delta y_{n}$, and $\Delta z_{n}$ & $1 \mathrm{~mm}$ standard deviation & & \\
\hline \hline
\end{tabular}

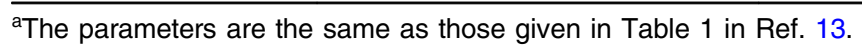

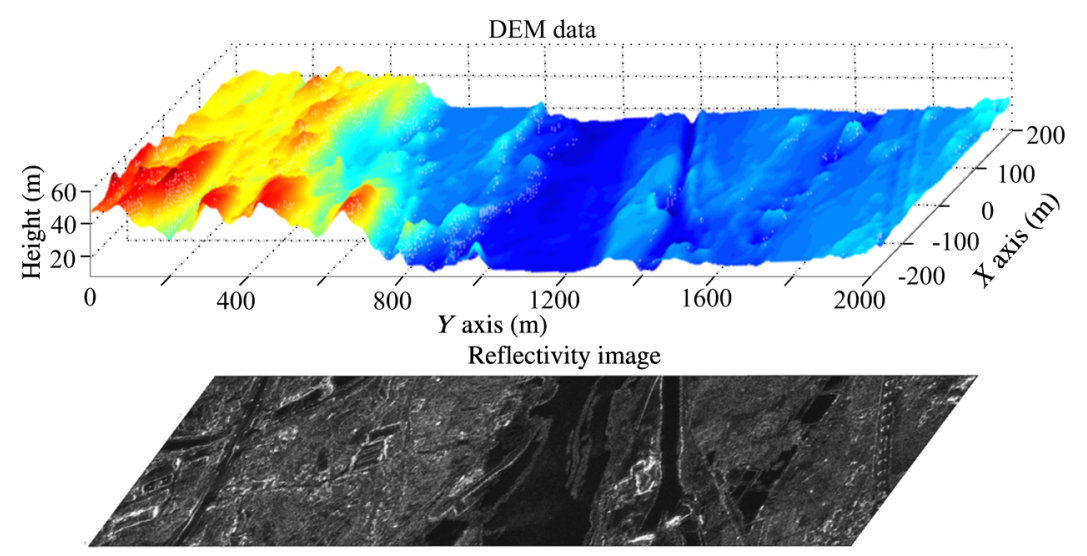

Fig. 13 3-D distributed target scene simulation input: DEM data and the corresponding 2-D reflectivity image.

Figure 14 shows the reconstructed 3-D image of the simulated target scene. The image with virtual element position errors is filled with strips of energy, which represent the cross-track degradation due to a high integrated sidelobe level, making the targets in the distributed scenario indiscernible. If we apply the proposed calibration method based on time-divided active calibrators, the position errors can be calibrated within a very high accuracy of $0.02 \mathrm{~mm}$, which is shown in Fig. 15, so that the reconstructed 3-D image with calibration exhibits a good performance in the same manner as the error-free case.

Subsequently, Monte Carlo simulations with 200 iterations are conducted to demonstrate the root mean square error (RMSE) of the estimator versus the SNR, as well as the number of calibrators. The results are given in Fig. 16. In Fig. 16(a), it can be seen that the estimator's accuracy improves with the increase of SNR, and the SNR with a value of $20 \mathrm{~dB}$ can keep the RMSE well below $0.02 \mathrm{~mm}$, satisfying the calibration requirement of the virtual element position error (with

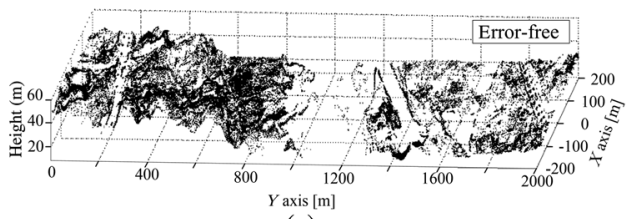

(a)

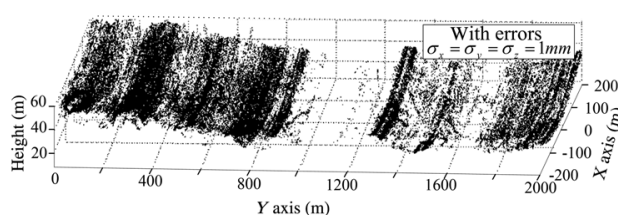

(b)

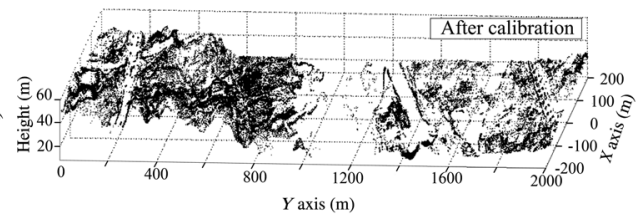

(c)

Fig. 14 Reconstructed 3-D images of the simulated target scene (displayed at the isosurface value of $9 \mathrm{~dB}$ ). 
Tan et al.: Array error calibration methods in downward-looking linear-array...

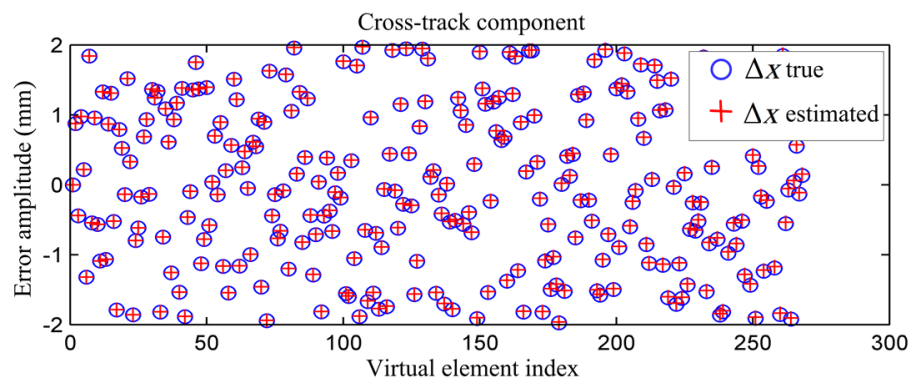

(a)

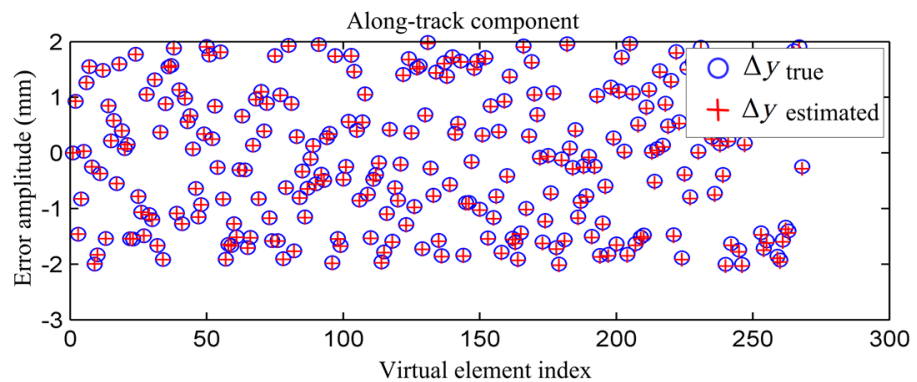

(b)

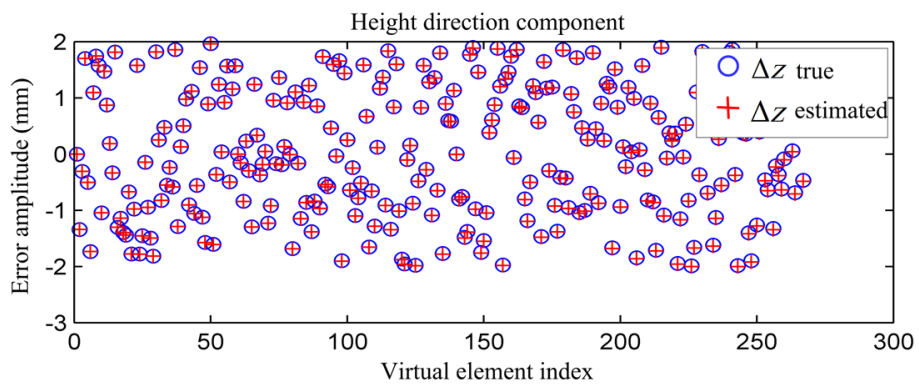

(c)

Fig. 15 The estimated values of the virtual element position errors in (a) cross-track direction, (b) along-track direction, and (c) height direction as compared to their respective true values.

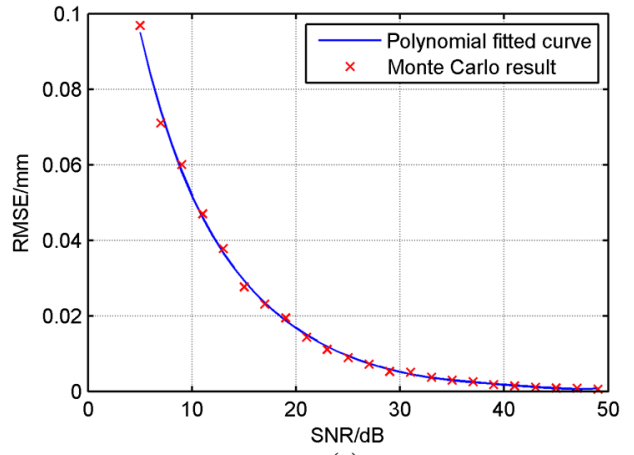

(a)

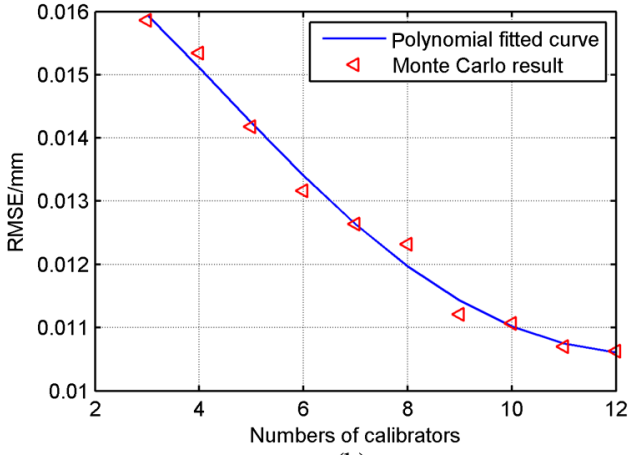

(b)

Fig. 16 RMSE versus (a) SNR and (b) number of calibrators $(S N R=20 \mathrm{~dB})$.

a standard deviation of $0.075 \mathrm{~mm}$ ). In Fig. 16(b), the RMSE values corresponding to different numbers of randomly distributed calibrators under a 20-dB SNR level are given. Though the trend of RMSE improvement with the increase of the calibrator's number is obvious, we can clearly see that using only three calibrators is quite enough for keeping the accuracy below the calibration requirement mentioned before. Consequently, using three calibrators is the cost-effective solution for the time being. 


\section{Conclusions}

The LA-3D-SAR is a coherent system and is particularly susceptible to array errors, such as the multichannel amplitude-phase errors and virtual element position errors. These errors are traceable to the nonideal electrical characteristics of antennas, vibration due to flight disturbance, and motion measurement errors. In this paper, the impact of the multichannel amplitude-phase errors and virtual element position errors on 3-D imaging quality with regard to their characteristics and level of severity is investigated and it has been shown that a small amount of error may cause massive degradations within the final 3-D SAR image. For the multichannel amplitude-phase errors, which increase the integrated sidelobe level in the array direction, the target response is more sensitive to the phase error than to the amplitude error. The amplitude error can be corrected by a simple power normalization while the phase error can be calibrated using external (parallel or point target) calibrators, as proposed and experimentally verified in the paper. For the virtual element position errors, it has been shown that a high integrated sidelobe level, which generates contrast degradation and noise can be caused in the cross-track direction, but with little impact on the range and along-track directions. By contrast, the cross-track beamforming performance is more sensitive to the error component in the height direction, and for typical Kaband LA-3D-SAR parameters, the tolerable level of the error standard deviation is found to be below the submillimeter level. To mitigate the error effects, calibration and compensation measures based on time-divided active calibrators are proposed and simulated in the paper. It is worth noting that in designing an LA-3D-SAR system, as well as in processing the LA-3D-SAR data, the analyses and investigations in this paper will provide a useful reference to specify the system performance, calibration requirements, and how to eliminate the error effects and optimize the imaging result.

\section{Acknowledgments}

This work is supported by the National Natural Science Foundation for Young Scientists of China (Grant Nos. 61201433 and 61401434), the National Natural Science Foundation Project (Grant No. 61372186), Program for Young Talents of Science and Technology in Universities of Inner Mongolia Autonomous Region (Grant No. NJYT-14-B09).

\section{References}

1. M. Soumekh, Synthetic Aperture Radar Signal Processing with Matlab Algorithms, Wiley, New York (1999).

2. J. Shi et al., "APC trajectory design for 'one-active' linear-array three-dimensional imaging SAR," IEEE Trans. Geosci. Remote Sens. 48, 1470-1486 (2010).

3. Y. Cao et al., "A mathematical model for MIMO imaging," Proc. SPIE 8393, 5 (2012).

4. P. Huang and W. Xu, "ASTC-MIMO-TOPS mode with digital beam-forming in elevation for high-resolution wide-swath imaging," Remote Sens. 7, 2952-2970 (2015).

5. W. Q. Wang, "Virtual antenna array analysis for MIMO synthetic aperture radars," Int. J. Antennas Propag. 2010(6), 1-10 (2012).

6. H. Z. Shao and W. Q. Wang, "Multiple-input multiple-output synthetic aperture radar antenna arrangement for wide-swath remote sensing," J. Appl. Remote Sens. 9, 094097 (2015).

7. J. C. Yang, W. M. Su, and H. Gu, "Two-dimensional superresolution multiple-input multiple-output and inverse synthetic aperture radar imaging based on spatial frequency ambiguity resolving," J. Appl. Remote Sens. 9(1), 095090 (2015).

8. X. Yang et al., "Amplitude and phase errors correction for array 3D SAR system based on single prominent point like target echo data," J. Radars 4, 409-418 (2014).

9. J. Klare, A. R. Brenner, and J. H. Ender, "A new airborne radar for 3D imaging-image formation using the ARTINO principle," in Proc. 6th European Conf. on Synthetic Aperture Radar, pp. 1-4, Aachen, Germany (2006). 
10. J. F. Nouvel et al., "ONERA DRIVE project," in Proc. European Conf. on Synthetic Aperture Radar, pp. 1-3 (2008).

11. W. Tan et al., "Synthetic aperture radar tomography sampling criteria and three-dimensional range migration algorithm with elevation digital spotlighting," Sci. China Ser. F: Inf. Sci. 52(1), 100-114 (2009).

12. X. Peng et al., "Airborne downward looking sparse linear array 3-D SAR heterogeneous parallel simulation,” Remote Sens. 5, 5304-5329 (2013).

13. K. Han et al., "Efficient pseudopolar format algorithm for down-looking linear-array SAR 3-D imaging," IEEE Geosci. Remote Sens. Lett. 12, 572-576 (2015).

14. X. Ren, L. Chen, and J. Yang, "3D imaging algorithm for down-looking MIMO array SAR based on Bayesian compressive sensing," Int. J. Antennas Propag. 2014, 1-9 (2014).

15. S. Zhang, Y. Zhu, and G. Kuang, "Imaging of downward-looking linear array three-dimensional SAR based on FFT-MUSIC," IEEE Geosci. Remote Sens. Lett. 12(4), 885-889 (2015).

16. J. Shi et al., "An optimal DEM reconstruction method for linear array synthetic aperture radar based on variational model," J. Radars 4(1), 20-28 (2015).

17. W. G. Carrara et al., Spotlight Synthetic Aperture Radar: Signal Processing Algorithms, Artech House, Boston, Massachusetts (1995).

18. J. Klare et al., "Image quality analysis of the vibrating sparse MIMO antenna array of the airborne 3D imaging radar ARTINO," in Proc. IEEE Int. Geosci. Remote Sens. Symp., pp. 5310-5314 (2007).

19. J. Klare, A. Brenner, and J. Ender, "Impact of platform attitude disturbances on the 3D imaging quality of the UAV ARTINO," in Proc. European Conf. on Synthetic Aperture Radar, pp. 1-4 (2008).

20. A. V. Oppenheim, Discrete-Time Signal Processing, 2/E, Pearson Education, India (2006).

21. K. Han et al., "Statistical analysis of the effects of virtual element position errors on airborne down-looking LASAR 3-D imaging," IEEE Geosci. Remote Sens. Lett. 12, 1-5 (2015).

22. W. Philips, "ECG data compression with time-warped polynomials," IEEE Trans. Biomed. Eng. 40, 1095-1101 (1993).

23. I. G. Cumming and F. H. Wong, Digital Signal Processing of Synthetic Aperture Radar Data: Algorithms and Implementation, Artech House, Norwood (2004).

24. J. R. Fienup, "Phase error correction for synthetic-aperture phased-array imaging systems," Proc. SPIE 4123, 47-55 (2000).

25. M. Zhang and Z.-D. Zhu, "DOA estimation with sensor gain, phase and position perturbations," in Proc. of the IEEE 1993 National Aerospace and Electronics Conf., pp. 67-69 (1993).

26. N. Fistas and A. Manikas, "A new general global array calibration method," in Proc. IEEE ICASSP-94 Conf., pp. IV/73-IV/76 (1994).

27. K. V. Stavropoulos and A. Manikas, "Array calibration in the presence of unknown sensor characteristics and mutual coupling," in 10th European Signal Processing Conf., pp. 1-4 (2000).

28. H. Krim and M. Viberg, "Two decades of array signal processing research: the parametric approach," IEEE Trans. Signal Process. Mag. 13, 67-94 (1996).

29. G. Bienvenu and L. Kopp, "Adaptivity to background noise spatial coherence for high resolution passive methods," in Int. Conf. on Acoustics, Speech, and Signal Processing, Vol. 5, pp. 307-310 (1980).

30. A. Freeman, "SAR calibration: an overview," IEEE Trans. Geosci. Remote Sens. 30, 11071121 (1992).

31. Y. Qixiao and S. Yonghuan, Practical Mathematics Handbook, Science Press, Beijing, China (2006).

32. G. Golub and C. V. Loan, Matrix Computations, 3rd ed., Johns Hopkins University Press (1996).

33. KeySight (Agilent Technologies), "PNA series network analyzers online help (User Guide, Programming Guide), a.10.40 ed.," http://na.support.keysight.com/pna/help/latest/help.htm (2015). 
34. W. Hong et al., "Development and experiments of ground-based SAR in IECAS for advanced SAR imaging technique validation," in Proc. European Conf. on Synthetic Aperture Radar, pp. 1-4, Aachen, Germany (2010).

35. Y. Lin et al., "Airborne circular SAR imaging: results at P-band," in Proc. IEEE Int. Geosci. Remote Sens. Symp., pp. 5594-5597 (2012).

Weixian Tan is an associate professor at the Inner Mongolia University of Technology, Hohhot, China. He received his $\mathrm{PhD}$ in signal and information engineering from the Institute of Electronics, Chinese Academy of Sciences, in 2009. He is the author of more than 50 journal and conference papers. His current research interests include microwave imaging, holography, and synthetic aperture radar systems.

Pingping Huang received his $\mathrm{PhD}$ from the Institute of Electronics, Chinese Academy of Sciences, Beijing, China, in 2010. He is currently with the College of Information Engineering, Inner Mongolia University of Technology, Hohhot, China. His current major research interests are signal processing, digital beamforming, polarimetric interferometry, and spaceborne synthetic aperture radar system design.

Kuoye Han received his PhD from the Institute of Electronics, Chinese Academy of Sciences, Beijing, China, in 2015. He is currently with the Information Science Academy, China Electronics Technology Group Corporation. His current major research interests are phased-array radar, synthetic aperture radar system design, digital beamforming, and microwave imaging.

Qi Liu is a senior engineer at China Institute of Water Resources and Hydropower Research, Beijing, China. She received her $\mathrm{PhD}$ in signal and information engineering from the Institute of Electronics, Chinese Academy of Sciences, in 2009. Her current research interests include microwave imaging, water information, and rain radar.

Xueming Peng received his bachelor's degree in electrical engineering and automation from Anhui University, Hefei, China, in 2009, and his PhD from the University of Chinese Academy of Sciences, Beijing, China, in July 2014. From August 2014 to May 2015, he was with the No. 802 Research Institute, Shanghai Academy of Spacelight Technology (also known as Shanghai Radio Equipment Institute). He is currently a researcher with the Global R\&D Centre (Shanghai), Carestream Health, Inc., Shanghai, China. His main research interests are radar signal processing, heterogeneous parallel computing and real-time operating systems, and 3-D polygon mesh processing. 